\title{
The $h A T$-family transposable element, hopper, from Bactrocera dorsalis is a functional vector for insect germline transformation
}

\author{
Alfred M. Handler ${ }^{1 *}$ and Marc F. Schetelig ${ }^{2}$
}

\begin{abstract}
Background: The hopper hAT-family transposable element isolated from the Oriental fruit fly, Bactrocera dorsalis, is distantly related to both the Drosophila hobo element and the Activator element from maize. The original $3120 \mathrm{bp}$ hopper $^{\text {Bd-Kah }}$ element isolated from the Kahuku wild-type strain was highly degenerate and appeared to have a mutated transposase and terminal sequences, while a second 3131 bp element, hopper ${ }^{\text {Bd-we }}$, isolated from a white eye mutant strain had an intact transposase reading frame and terminal sequences consistent with function.

Results: The hopper ${ }^{\text {Bd-we }}$ element was tested for function by its ability to mediate germline transformation in two dipteran species other than $B$. dorsalis. This was achieved by creating a binary vector/helper transformation system by linking the hopper ${ }^{\text {Bd-we }}$ transposase reading frame to a D. melanogaster hsp70 promoter for a heat-inducible transposase helper plasmid, and creating vectors marked with the D. melanogaster mini-white ${ }^{+}$or polyubiquitinregulated DsRed fluorescent protein markers.

Conclusions: Both vectors were successfully used to transform D. melanogaster, and the DsRed vector was also used to transform the Caribbean fruit fly, Anastrepha suspensa, indicating a wide range of hopper function in dipteran species and, potentially, non-dipteran species. This vector provides a new tool for insect genetic modification for both functional genomic analysis and the control of insect populations.
\end{abstract}

Keywords: Insect genetic modification, Transposon-mediated transformation, Tephritidae

\section{Background}

Transposon-mediated germline transformation has been the primary method of insect genomic manipulation since a $P$ element vector was successfully transposed into the Drosophila melanogaster genome [1]. More recent gene-editing techniques, such as CRISPR/Cas9, have provided additional methods for genome manipulation, but thus far are limited in achieving genomic

\footnotetext{
*Correspondence: al.handler@usda.gov

'USDA/ARS, Center for Medical, Agricultural and Veterinary Entomology, 1700 SW 23rd Drive, Gainesville, FL 32608, USA

Full list of author information is available at the end of the article
}

integration of DNA constructs greater than several kilobases [2]. This limitation is especially critical for the development of genetically modified strains to improve biologically-based strategies for the control of insect populations harmful to agriculture and human health, for which conditional lethal and other transgene constructs are typically $10 \mathrm{~kb}$ or greater. Significantly, geneediting is also limited in generating random genomic insertions for mutagenesis and enhancer-trap screens that have proven highly advantageous for functional genomic analysis, most clearly demonstrated by elucidating gene function and regulation in the D. melanogaster model 
system. Thus, the ability to use transposon vector systems such as the $P$ element in a wide range of insect systems has been, and remains, a high priority for understanding and manipulating insect genomes.

Unfortunately, $P$ element mobility was found to be atypically limited to the Drosophila genus and closely related species and has never been successfully used to achieve transposon-mediated transformation in a nondrosophilid species [3]. More than a decade passed before other Class II transposable elements were discovered that were functionally less restricted and successful in achieving non-drosophilid transformation, which included mariner/Mos 1 discovered in D. mauritiana that initially transformed Aedes aegypti [4, 5], Minos discovered in D. hydei that initially transformed Ceratitis capitata [6, 7], piggyBac from the cabbage looper moth, Trichoplusia $n i$, that also initially transformed C. capitata [8, 9], and the Hermes element found in Musca domestica [10] used initially to transform Ae. aegypti [11]. Hermes is a member of the hobo, Activator, Tam3 ( $h A T)$ family of transposons that has been found to be phylogenetically widespread [12]. While this supports the notion of widespread functionality, most full-length $h A T$ elements (having a coding region and at least one terminal repeat sequence) were found to be defective, and thus far none, other than Hermes, have been shown to support insect transformation.

One of the defective $h A T$ elements, hopper ${ }^{\text {Bd-Kah }}$, was originally discovered by use of a short $h A T$-related PCR product as a hybridization probe to a lambda GEM12 genome library of the Kahuku wild type strain of the Oriental fruit fly, Bactrocera dorsalis [13]. The sequenced element was found to be $3120 \mathrm{bp}$ in length with $19 \mathrm{bp}$ terminal inverted repeat (TIR) sequences with a single mismatch. The TIRs surrounded a $1.9 \mathrm{~kb}$ consensus $h A T$ transposase transcriptional unit that was, however, interrupted by two frameshift mutations consistent with it being non-functional, in addition to a degenerate 8-bp genomic insertion site duplication. Southern blots to genomic DNA from several B. dorsalis strains, and those from the melon fly, Zeugodacus cucurbitae, showed the presence of elements highly conserved with hopper $^{\mathrm{Bd}-\mathrm{Kah}}$ indicating the existence of related elements in, and possibly beyond, the genus.

In an effort to discover a functional paralog of hopper ${ }^{\mathrm{Bd}-\mathrm{Kah}}, 5^{\prime}$ and $3^{\prime}$ primers to the hAT element were used in opposite orientation for inverse PCR in one of the other $B$. dorsalis strains carrying a white eye (we) mutation and the presence of elements similar to hop$p^{\text {Bd-Kah }}[14,15]$. This led to the discovery of a new 3131 bp element in B. dorsalis, hopper ${ }^{\text {Bd-we }}$. Unlike hopper $^{\text {Bd-Kah }}$, hopper ${ }^{\text {Bd-we }}$ was found to have an uninterrupted 1950 bp reading frame, intact 19-bp TIR and 16bp sub-TIR sequences, and a duplicated 8-bp insertion site sequence consistent with a functional transposon. While this full-length element provided insights into the conserved functional domains for Class II elements in general, and $h A T$ elements specifically, its ability to autonomously transpose (requiring functional transposase and requisite terminal sequences) remained unknown. Function for hopper ${ }^{\text {Bd-we }}$ was implied by preliminary embryonic transposon mobility assays in $B$. dorsalis, however, function for the $D$. melanogaster hobo hAT element was also implied for five tephritid species using simlar assays [16], yet in our hands it was never successful in achieving non-drosophilid germline transformation. Thus, we initiated more direct and conclusive tests for hopper ${ }^{\text {Bd-we }}$ function based on its ability to mediate in vivo germline transformation in two dipteran species, D. melanogaster and A. suspensa.

\section{Results}

\section{Transformation experiments}

In the first of three transformation experiments we tested the hopper ${ }^{\mathrm{Bd}-\mathrm{we}}$ transposon vector system (now referred to as hopper) in the D. melanogaster $w[\mathrm{~m}]$ white (eye) mutant host strain using the hopper vector, pKhop $\left[\right.$ Dmwhite $\left.^{+}\right]$, marked with the $D$. melanogaster wild type mini-white ${ }^{+}$gene. This serves as a transformant mutant-rescue marker by complementing the host strain white mutation, resulting in eye pigmentation in $\mathrm{G}_{1}$ germline transformants. The marker insertion within the transposase coding region creates a nonautonomous vector that relies on an exogenous source of transposase for transposition, that was provided by the pUChsHopper helper plasmid having the transposase gene under $D$. melanogaster $h s p 70$ promoter regulation for induction of expression by heat shock [17]. A mixture of vector and helper plasmids was injected into 436 dechorionated eggs under halocarbon oil, from which $131 \mathrm{G}_{0}$ adults survived (Table 1). A total of 70 adults were backcrossed individually to $w[\mathrm{~m}]$ adults, with an additional $61 \mathrm{G}_{0}$ adults mated in 28 groups of 2 to 3 to $w[\mathrm{~m}]$ adults of the opposite sex, for a total of $98 \mathrm{G}_{0}$ lines. Seventy-nine $G_{0}$ fertile matings were screened for $\mathrm{G}_{1}$ progeny with pigmented eyes that were observed in two of the matings, F11A and F23A (Fig. 1a), resulting in a transformation frequency of $2.5 \%$ per fertile $G_{0}$. The F11A $G_{0}$ mating yielded three transformant progeny that were considered to be $G_{1}$ siblings resulting from the same transformation event. It is possible for marked $G_{1}$ siblings to arise from independent vector insertions, however all three lines shared the same female-specific marker phenotype that was mapped to the $\mathrm{X}$ chromosome in F11A, consistent with a common germline transformation event (see below).

The second hopper transformation was similarly performed in the Drosophila $w[\mathrm{~m}]$ strain, but using the 


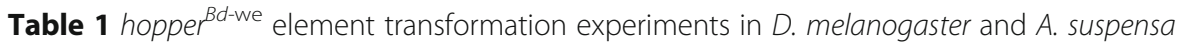

\begin{tabular}{llllllll}
\hline Host $^{\mathbf{a}}$ & Vector & $\mathbf{G}_{\mathbf{0}}$ eggs injected & $\mathbf{G}_{\mathbf{0}}$ s mated $^{\mathbf{b}}$ & No. $\mathbf{G}_{\mathbf{0}}$ lines & No. fertile $\mathbf{G}_{\mathbf{0}}$ lines & $\begin{array}{l}\text { No. } \mathbf{G}_{\mathbf{1}} \\
\text { transformant lines }\end{array}$ & $\begin{array}{l}\text { Transformation } \\
\text { frequency }\end{array}$ \\
\hline Dm & pKhop [Dmwhite+] & 436 & 131 & 98 & 79 & 2 & 0.025 \\
Dm & phop [PUb-DsRed.T3] & 1056 & 203 & 170 & 129 & 2 & 0.016 \\
As & phop [PUb-DsRed.T3] & 1453 & 389 & 103 & 94 & 9 & 0.096 \\
\hline
\end{tabular}

${ }^{\mathrm{a}} \mathrm{Dm}$ : D. melanogaster $w[\mathrm{~m}]$ strain; $A s: A$. suspensa wild type strain

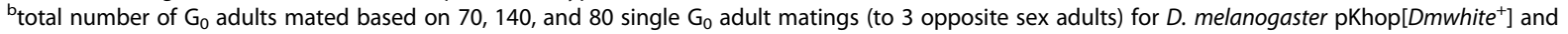
phop[PUb-DsRed.T3] transformations, and the A. suspensa phop[PUb-DsRed.T3] transformation, respectively; additional $\mathrm{G}_{0} \mathrm{~s}$ were mated in groups of $2-3$ same sex $\mathrm{G}_{0}$ adults to opposite sex adults

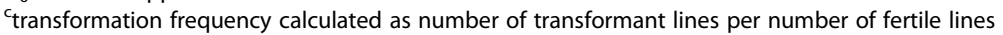

phop[PUbDsRed.T3] vector, marked with a strongly expressing fluorescent DsRed variant [18]. For this experiment 1056 eggs were injected, from which $203 G_{0}$ adults survived that were backcrossed to $w[\mathrm{~m}]$ in 170 matings (Table 1). From 129 fertile $G_{0}$ matings, $G_{1}$ transformant progeny expressing full-body DsRed fluorescence from 2 independent matings (F73A and F74A) were observed (Fig. 1b), yielding a transformation frequency of $1.6 \%$ per fertile $G_{0}$. Similar to the F11A line, F74A yielded four sib transformants that also exhibited female-specific marker expression that was X-linked.

The third hopper transformation also tested the phop[PUbDsRed.T3] vector, but in the Caribbean fruit fly, $A$. suspensa, that is in the same Tephritidae family as $B$. dorsalis. In this experiment 1453 eggs were injected from which 389 adults survived that were backcrossed to wild type $A$. suspensa in 80 individual $G_{0}$ matings and 23 group matings (Table 1 ). From 94 fertile $G_{0}$ matings, $\mathrm{G}_{1}$ DsRed transformant progeny from 9 independent matings (lines F2M, F5M, F7, F30, F36, M8A, M31, M32, and M35) were observed (Fig. 1c), yielding a transformation frequency of $9.6 \%$ per fertile $G_{0}$. All of the lines, except for F5M, expressed DsRed in both male and female adults that presumably resulted from autosomal integrations. Fluorescent marker expression in F5M, however, was only observed in male progeny that would imply a Y-linked insertion.

\section{Verification and molecular analysis of genomic hopper transformant integrations}

To verify that transformant lines from each experiment were the result of genomic germline transposonmediated vector integrations, $5^{\prime}$ and $3^{\prime}$ sequences flanking the hopper vector were isolated by TAIL-PCR or inverse PCR and sequenced (Fig. 2 and Additional file 1 Figure S1). Transposon-mediated vector genomic integrations were indicated by intact terminal sequences of the hopper vector, but having duplicated 8-bp insertion sites and flanking sequences that differed from hop$p r^{\text {Bd-we }}$ and its insertion site sequences in the vector plasmid (Fig. 2a), eliminating the possibility of vector integration by a recombinant event. Additional evidence was provided for some transformations whose genomic insertion sites could be identified and mapped by BLASTn database searches.

For the D. melanogaster $\mathrm{pKhop}\left[\right.$ Dmwhite $\left.^{+}\right]$F11A transformant line, TAIL-PCR yielded 221-bp for the 5' flanking sequence and 151-bp for the 3' flanking sequence having nearly $98 \%$ identity with the $\mathrm{X}$ chromosome-linked CG34339/Megalin $141 \mathrm{~kb}$ gene from a BLASTn search of the $D$. melanogaster NCBI database (Fig. 2b and Additional file 1 Figure S1a). The 8-bp duplicated insertion site sequence was GTGCTGCG compared to the hopper vector CTAAAAAA duplicated insertion site isolated from the $B$. dorsalis white eye strain genome. Notably, mutant-rescue transformants expressing red eye pigmentation were limited to females suggesting that the vector insertion created an X-linked recessive lethal mutation, that was supported by a backcross of F11A females to $w[\mathrm{~m}]$ males. This resulted in $111 \mathrm{~F}_{1}$ white-eye males and 203 females that were $48 \%$ red eye that is consistent with an $\mathrm{X}$-linked recessive lethal based upon male/female survival, marker phenotype segregation, and with previously created X-linked mutations in the Megalin locus [19].

For the D. melanogaster $\mathrm{pKhop}\left[\right.$ Dmwhite $\left.^{+}\right] \mathrm{F} 23 \mathrm{~A}$ transformant line, TAIL-PCR yielded 328-bp for the 5' flanking sequence and 884-bp for the 3' flanking sequence. A D. melanogaster BLASTn search showed that this sequence has greater than $98 \%$ identity to the chromosome 2 intergenic region between the septin interacting protein 2 (sip2) and coproporphyrinogen oxidase (coprox) genes at the 27C7 locus (Fig. 2c and Additional file 1 Figure S1b). The 8-bp duplicated insertion site sequence was GTTGTAAC.

For the D. melanogaster phop[PUbDsRed.T3] F74A transformant line, TAIL-PCR yielded 1017-bp for the $5^{\prime}$ flanking sequence and 102-bp for the 3' flanking sequence. A D. melanogaster BLASTn search showed that this sequence has nearly $97 \%$ identity to the first intron of the X chromosome-linked ocelliless (oc) gene at locus 7F10-8A1 (Fig. 2d and Additional file 1 Figure S1c). The 8-bp duplicated insertion site sequence was GTCAGG AG. An interesting aspect of this hopper integration is that, similar to the F11A line, transformants expressing DsRed fluorescence were limited to females consistent 


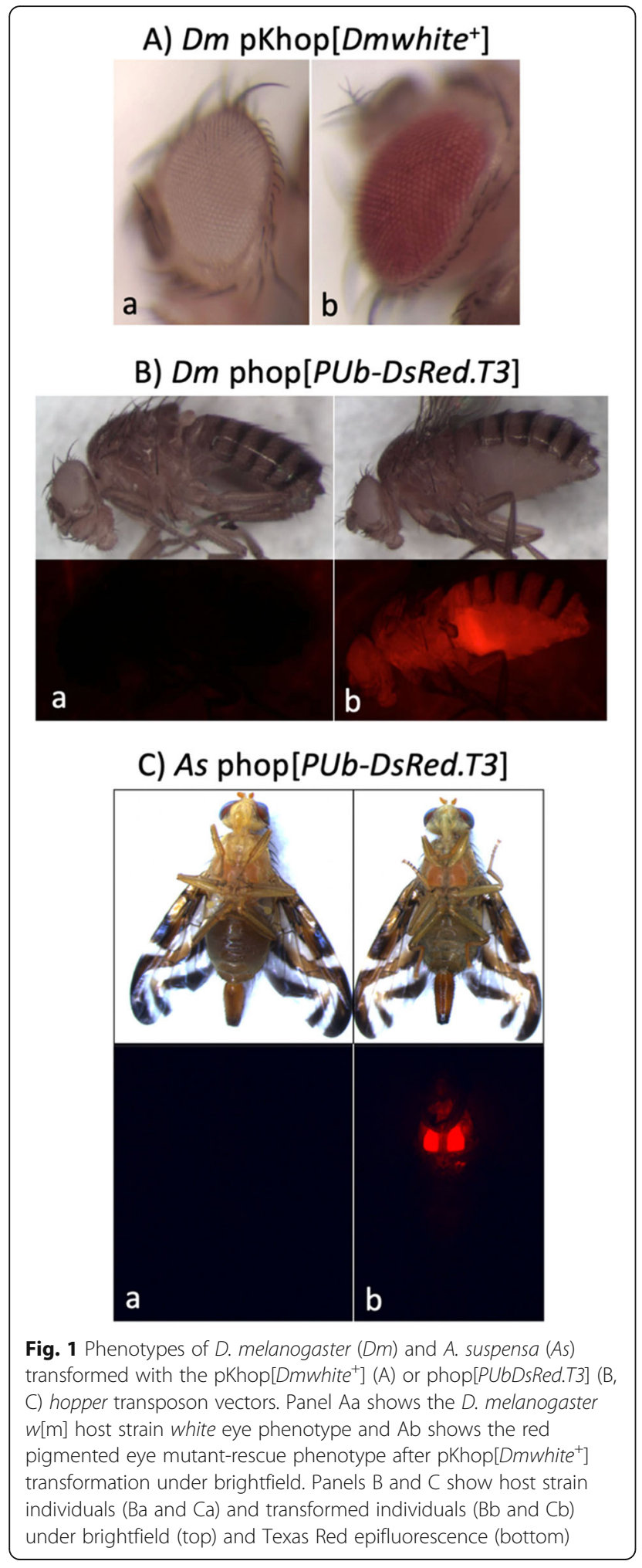

with the creation of an X-linked recessive lethal mutation. Segregation analysis of a backcross of F74A females to $w[\mathrm{~m}]$ males resulted in 76 non-fluorescent $\mathrm{F}_{1}$ males and 140 females that were $42 \%$ DsRed fluorescent consistent with an X-linked recessive lethal based upon male/female survival and marker phenotype segregation. However, while $o c$ mutations cause neural defects that result in the absence of the ocelli photoreceptors in homozygous mutants, homozygotes remain recessive viable, though an allele of the polycistronic oc locus, orthodenticle (otd), has a moderate dominant phenotype and is recessive lethal [20]. Thus, the vector insertion appears to be more highly related to the otd phenotype rather than $o c$. Another consideration for this insertional mutation is that it occurs in the $o c$ first intron, that would typically be considered silent, though an otd enhancer has been identified in intron 3 [21] and conceivably another otd enhancer or regulatory region might exist in intron 1 for this complex locus.

For the A. suspensa phop[PUbDsRed.T3] M8A transformant line, TAIL-PCR yielded 946-bp for the $5^{\prime}$ flanking sequence and 262-bp for the 3' flanking sequence (Fig. 2e and Additional file 1 Figure S1d), but was inconclusive for the other Anastrepha transformant lines. However, inverse PCR of line F2M yielded genomic sequences of 73-bp at the $5^{\prime}$ junction and 861-bp at the 3' junction (Fig. $2 \mathrm{f}$ and Additional file 1 Figure S1e). A high quality assembly for the A. suspensa genome is currently unavailable, and a BLASTn search of the NCBI nr/nt database, as well as the D. melanogaster and Ceratitis capitata (medfly) databases, found no significant identities for the genomic insertion site of either transformant line. However, the two insertion site sequences did share the same 8-bp duplicated GAACAAAT insertion site sequence, and there was high identity between the adjacent 37-bp sequences at the $5^{\prime}$ junction. Considering the highly repetitive $\mathrm{A} / \mathrm{T}$ content for both insertion sites (Additional file 1 Figure S1d,e), 83\% for M8A and 66\% for F2M, it may be assumed that the insertions occurred in species-specific non-coding regions, and highly repetitive regions may have hindered the PCR isolation of insertion sites for the other lines.

\section{Discussion}

The hopper ${ }^{\mathrm{Bd}-w e}$ transposable element previously discovered in the tephritid fruit fly, B. dorsalis, has been shown to function as a transposon vector for germline transformation in two other dipteran species, consistent with it being an autonomously functional element. Relatively modest 1.6 and $2.5 \%$ transformation frequencies were achieved with two independently marked vectors in $D$. melanogaster, using mutant-rescue restoration of eye pigmentation as a marker for one vector, and ectopic DsRed fluorescent protein expression as marker for another. The latter vector also achieved transformation in the tephritid fruit fly, A. suspensa, which is more closely related to $B$. dorsalis, at a higher rate of $9.6 \%$ per fertile 
a) B. dorsalis hopper ${ }^{\text {Bd-we }}$ chromosomal insertion site GAAAAAATGTGGGCGCTAAAㅗㅅㅗ $<<5$ ' - hopper ${ }^{\text {Bd-we }}-3$ ' $>$ CTAAA소고TATTTATTCTCACAG

b) D. melanogaster line F11A - phop[Dmwhite $\left.{ }^{+}\right]$vector Blast Hit: CG34339/Megalin (X chromosome; female-specific putative recessive lethal)

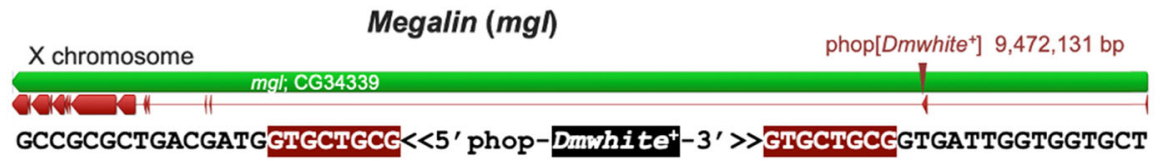

c) D. melanogaster line F23A - phop[Dmwhite $\left.{ }^{+}\right]$vector Blast Hit: septin interacting protein 2 (sip2)

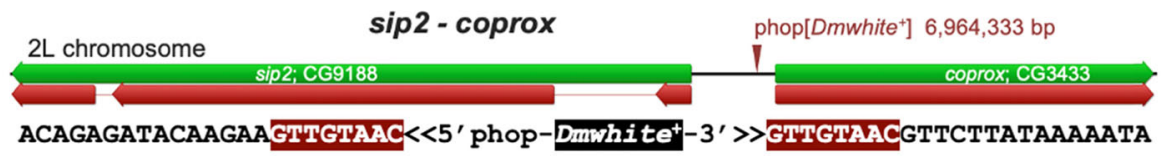

d) D. melanogaster line F74A - phop[PUbDsRed.T3] vector

Blast Hit: ocelliless (oc); also known as orthodenticle (otd) (X chromosome; female-specific putative recessive lethal)

Ocelliless

\section{e) A. suspensa line M8A - phop[PUbDsRed.T3] vector Blast Hit: none}

ATACAGGAAAATAAAGACANAT< $<5$ ' phop-PUbDsRec-3' $>$ GAACAAMATGCATAGTGACCTT

\section{f) A. suspensa line F2M - phop[PUbDsRed.T3] vector Blast Hit: none \\ ATACAAGAAAATAAAGACAAMT< $<5$ ' phop-PUbDsRec-3' $>$ GACAMATAGTGACCTTCAGACT}

Fig. 2 Flanking genomic insertion site sequences for the B. dorsalis hopper ${ }^{B d-w e}$ transposable element in the $B$. dorsalis white eye strain genome (a) compared to pKhop [Dmwhite ${ }^{+}$and phop[PUbDsRed.T3] vector insertions in D. melanogaster (b-d) and A. suspensa (e-f). Twenty-three (23) nucleotide $5^{\prime}$ and $3^{\prime}$ flanking genomic sequences shown for vector insertions in the designated transformant lines, including the proximal 8-bp duplicated insertion site sequence (brown outline). The highest identity Blastn hit (>95\%) for the complete vector insertion site sequences is provided (see Additional file 1: Fig. S1 for complete insertion site sequences). Below flanking genomic sequences (b-d) is a schematic diagram of the genomic insertion site position (annotations: green, gene; red, exons; arrow, insertion site position)

$G_{0}$, however additional experiments in these and related dipteran species will be necessary to determine if there is a significant species-specific relationship to transpositional frequency. Though not shown to be a highly robust vector in this initial study, hopper yielded transformants in each individual experiment, and most of the transformant lines created in this study have been cultured in the laboratory for a minimum of 6 to 8 years. Thus, hopper appears to be a reliable and stable vector for these two species, but as with all newly discovered vectors, actual transformation efficacy and range of function will depend upon continued tests in insects from several Orders. Since the functional element exists in $B$. dorsalis, and a closely related (if not the same) hopper element exists in the closely related species, $Z$. cucurbitae [14], its ability to routinely create stable transformants in these and related tephritids also remains to be evaluated. 
Beyond the possibility for differential transpositional activity in the two species, the limited number of insertions molecularly characterized, nevertheless, raise the possibility for preferential insertion sites. For D. melanogaster, the three transformant lines analyzed resulted from vector insertions within actively expressing genes (including exons and an intron), or within a short intergenic sequence. In contrast, both hopper vector insertions in A. suspensa were localized to highly repetitive $\mathrm{A} / \mathrm{T}$ enriched non-coding regions. Furthermore, both insertions targeted identical 8-bp GAACAAAT genomic sites having an additional 37-bp sequence identity at the $5^{\prime}$ junction. Transposon vector insertions in the same variable target site sequence do occur, if not the same genomic target site, but these have usually been discovered in large screens of a re-mobilized transposon [22, 23]. In addition, two of the four Drosophila transformations were putative X-linked recessive lethal insertions, and one of the nine Anastrepha transformations was a putative Y-linked insertion. While sex-linked insertions are occasionally found in transformations with other vectors in various species, we are not aware of a bias for this type of insertion site preference. Together, the findings from this initial limited study of hopper transpositions are intriguing that will require an expanded analysis for verification.

One of the benefits of transposon-mediated transformation not afforded by other types genetic manipulation is their ability to randomly insert into the genome that allows transposons to create mutations by insertional $\mathrm{mu}$ tagenesis, or be used as 'traps' to identify and isolate enhancers [24], proteins/exons [25, 26] and promoters [23]. These methods are of particular interest in species where newly identified genetic reagents, such as a variety of promoters and genes affecting viability and sex determination, are essential for genetic modifications that improve population control systems such as SIT. However, these transposon-based tools typically rely on the postintegration remobilization of the requisite vector for each system, and not all transposons are subject to remobilization in all species, possibly due to speciesspecific transpositional silencing. Most notable for this effect is the yellow fever mosquito, Aedes aegypti, that has been transformed with several vectors that have been completely, or highly, refractory to re-mobilization $[27,28]$. Thus, new transposon vectors increase the possibility for developing more effective re-mobilization systems, and also increases the means to create secondary transformations in transgenic strains without remobilizing the primary integration.

For more straightforward genomic insertions of gene constructs for comparative gene expression and other functional studies, and strain modification for pest biological control or enhanced fitness for beneficial insects
[29], transposon-mediated transformation is, currently, a more efficient if not more reliable process than the use of CRISPR/Cas9-mediated integration. Gene-editing based insertions, depending on homology-directed repair donor templates, are generally size-limited to a few thousand base pairs [2], while many constructs for strain modification are $10-15 \mathrm{~kb}$ in length, with off-target modifications most easily minimized in species having a high quality whole genome sequence [30]. In addition, while efficient DNA delivery remains an impediment to germline transformation for many species, this process can also be a limitation for CRISPR/Cas9 modification, and it is notable that development of several CRISPR/ Cas9-mediated gene drive systems rely on transposonmediated transformation to create 'helper' strains with a genomic source of Cas enzyme [31].

Beyond the advantages that a new functional transposon vector may have for functional analysis and transgenic strain modification, it is also recognized that functional Class II transposons are agents of evolutionary change resulting from insertional mutagenesis [32], and a comparison of variations in non-functional defective elements in closely and distantly related species provides insights into their phylogenetic relationship [33]. Indeed, we already know that hopper is currently the most distantly related insect $h A T$ element to $D$. melanogaster hobo, being equidistant to Activator in maize, and that elements closely related to hopper ${ }^{\text {Bd-Kah }}$ exist in the Bactrocera species, Z. cucurbitae [13]. Thus, a more extensive survey of hopper elements in $B$. dorsalis and $Z$. cucurbitae, and tephritid species in general, may contribute to resolving their evolutionary relationships [34], and better define the limits for the practical use of hopper vectors for genetic modification.

\section{Conclusions}

The previously isolated hopper ${ }^{\text {Bd-we }}$ transposable element was verified as an autonomous functional element by its ability to mediate genomic transpositions in the germline of two dipteran species. This is the second insect $h A T$ element, in addition to Hermes, known to be functional in a non-drosophilid species thereby expanding the tools available for genetic modification and genomic functional analysis in these insect species, and possibly others. The discovery of both a degenerate defective and functional hopper element in $B$. dorsalis, that might also exist in $Z$. cucurbitae, suggests that this distantly related $h A T$ element has had a long history in the Bactrocera genus and may be instrumental in clarifying its phylogenetic complexity.

\section{Methods}

Insect rearing

The Drosophila melanogaster $w[\mathrm{~m}]$ strain and transformant lines were maintained at $25^{\circ} \mathrm{C}$ and reared under 
standard laboratory conditions [35, 36]. An inbred wild type colony of Anastrepha suspensa (Homestead, Florida) and transformant lines were also reared under standard laboratory conditions at $27^{\circ} \mathrm{C}$ and $60 \%$ humidity on a $12 \mathrm{~h}$ light:12 $\mathrm{h}$ dark cycle [37].

\section{Vector and helper plasmids}

pUChsHopper. The hsp70-regulated hopper ${ }^{\text {Bd-we }}$ transposase helper plasmid, pUChsHopper, was created by first isolating the hopper ${ }^{\mathrm{Bd}-\mathrm{we}}$ transposase coding region and polyA termination sequence as a blunt-EcoRV/XbaI fragment from $\mathrm{pBShopper}^{\mathrm{Bd}-\mathrm{we}}$ [14]. The hopper ${ }^{\mathrm{Bd}-w e}$ fragment was then ligated into a BamHI-blunted/XbaI site of pUChsRB, downstream of the 457-bp XbaI-XmnI $5^{\prime}$ non-translated sequence from $D$. melanogaster hsp 70 [17].

pKhop $\left[\right.$ Dmwhite $\left.{ }^{+}\right]$. The $\mathrm{pKhop}\left[\right.$ Dmwhite $\left.{ }^{+}\right]$vector was created by isolating a $4.2-\mathrm{kb}$ EcoRI-blunted fragment of the D. melanogaster mini- white $^{+}$cDNA gene [38] and ligating it into the pKhopper ${ }^{\mathrm{Bd}-\mathrm{we}}$ blunt-HincII site at $\mathrm{nt}$ 922 within the open reading frame, thereby eliminating functional transposase.

phop[PUbDsRed.T3]. The hopper ${ }^{\text {Bd-we }}$ vector marked with $D$. melanogaster polyubiquitin (PUb)-regulated DsRed.T3 [18] was created by isolating PUbDsRed.T3 from pBXLII [PUbDsRed.T3] as a blunt-SmaI /SacII 3.0$\mathrm{kb}$ fragment ligated into the blunt-SnaBI/SacII deletion in pBShopper ${ }^{\text {Bd-we }}$ [14].

\section{Transformation experiments}

Pre-blastoderm embryo injections for germline transformation were performed as described for D. melanogaster [36] and A. suspensa [37], but using DNA mixtures having vector/helper concentrations in injection buffer ( $5 \mathrm{mM} \mathrm{KCl} ; 0.1 \mathrm{mM}$ sodium phosphate $\mathrm{pH} 6.8$ ) of of

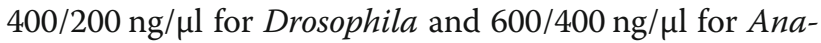
strepha. Dechorionated embryos were injected under Halocarbon 700 oil, placed within an oxygenated chamber for 16 to $20 \mathrm{~h}$ and subjected to a $37^{\circ} \mathrm{C}$ heat shock for $45 \mathrm{~min}$, and then reared at $25^{\circ} \mathrm{C}$ until adult eclosion. Eclosed $G_{0}$ adults were mated either individually to two or three $w[\mathrm{~m}]$ adults, or in groups of two to three $\mathrm{G}_{0}$ males or females backcrossed to $w[\mathrm{~m}] . \mathrm{G}_{1}$ eggs were collected for 10 to 14 days and reared under standard conditions. Putative transformant $G_{1}$ adults were screened for either eye pigmentation under bright field for pKhop $\left[\right.$ Dmwhite $\left.{ }^{+}\right]$injected flies, or whole body (though primarily thoracic flight muscle) DsRed fluorescence for phop[PUbDsRed.T3] injected flies. DsRed epifluorescence was observed under a Leica MZ FLIII microscope using a Texas Red filter set (ex: 560/40; em: 610 LP; Chroma).
Isolation of transgene integration flanking site sequences Flanking genomic sequences of $D m_{-}$F11A, Dm_F23A, $D m_{-}$F74A and As_M8A transgene integrations were isolated by TAIL PCR as described by Liu and Whittier [39], starting with $300 \mathrm{ng}$ of genomic DNA in the primary PCR reaction. The Platinum Taq Polymerase (Thermo Fisher) was used for all PCR reactions in a total volume of $25 \mu \mathrm{l}$ per reaction. Oligos used for the isolation of the 5' hopper vector insertion-site flanking sequences by TAIL PCR were the degenerate primer AD3 (AGWGNAGWANCAWAGG) and the hopper-specific primers R1_5hop_P952 (ACATTTGCTGAATATAAT ACCATTTACTTG), R2_5hop_P953 (GATATCTACT TGCATAAAATCATTCATTCG), and R3_5hop_P954 (ACTATCGAATGAATGAAAATTGCTGAAC). Oligos for the isolation of 3' hopper flanking sequence were the degenerate primer AD3 (AGWGNAGWANCAWAGG) and the specific primers F1_3hop_P955 (ACCTCGAT ATACAGACCGATAAAACACATGC), F2_3hop_P957 (CGTACGTCACAATATGATTATCTTTCTAGG), and F3_3hop_P958 (TCATTCAGTCATTAACAATCGATA GTTG). For the As_F2M transgene integration, the flanking genomic sequences were isolated by inverse PCR using HaeIII to digest the $5^{\prime}$ junction and MspI to digest the 3' junction. The circularized insertion sites were then isolated and sequenced using the primers P967_For (TGAAATTAAGCAGGTTGGCAACTTG) and P952_Rev (ACATTTGCTGAATATAATACCATT TACTTG) for the 5' junction, and P969_For (CGTT GTGACAAATAGTTTTTGCTTCC) and P956_Rev (TTGTTGTTTGAAGAGCACGCCTTTGC) for the 3' junction.

\section{Supplementary Information}

The online version contains supplementary material available at https://doi. org/10.1186/s12863-020-00942-3.

\section{Additional file 1.}

\section{Abbreviations}

AVT: Adenosine/Thymidine; Bd-Kah: Bactrocera dorsalis-Kahuku; Bd-we: Bactrocera dorsalis-white eye; BLASTn: Basic Local Alignment Search Tool nucleotide; coprox: coproporphyrinogen oxidase; CRISPR/Cas9: Clustered Regularly Interspaced Short Palindromic Repeats/CRISPR-associated 9 gene; hAT: hobo, Activator, Tam3; hsp70: D. melanogaster heat shock protein 70; NCBI: National Center for Biotechnology Information; oc. ocelliless; otd: orthodenticle; PCR: Polymerase chain reaction; phop [PUbDsRed.T3]: Plasmid hopper [polyubiquitin-DsRed.T3]; pKhop [Dmwhite ${ }^{+}$: Plasmid kanamycin-resistant hopper [D. melanogaster white ${ }^{+}$; sip2: septin interacting protein 2; TAlL-PCR: Thermal asymmetric interlaced PCR; TIR: Terminal inverted repeat

\section{Acknowledgements}

Grateful appreciation is extended to Robert Harrell II, Jennifer Mestas and Shelley Olson for assistance with the transformation experiments and transformant analysis. This study benefitted from discussions at International Atomic Energy Agency funded meetings for the Coordinated Research Project "Comparing Rearing Efficiency and Competitiveness of Sterile Male Strains Produced by Genetic, Transgenic or Symbiont-based Technologies". 


\section{About this supplement}

This article has been published as part of BMC Genetics Volume 21 Supplement 2, 2020: Comparing rearing efficiency and competitiveness of sterile male strains produced by genetic, transgenic or symbiont-based technologies. The full contents of the supplement are available online at https://bmcgenet. biomedcentral.com/articles/supplements/volume-21-supplement-2.

\section{Authors' contributions}

$\mathrm{AMH}$ created vector and transposase helper plasmids and designed, performed, and analysed data for transformation experiments; MFS designed, performed and analysed data for transgene vector insertion sites; and $\mathrm{AMH}$ and MFS wrote the manuscript. The authors read and approved the final manuscript.

\section{Funding}

Funding was provided by the USDA-NIFA-AFRI Foundational Program Grant \#2016-67013-25087 (to AMH) and the Emmy Noether program of the German Research Foundation (SCHE 1833/1-1 (to MFS). Publication costs were funded by the Joint FAO/IAEA Division of Nuclear Techniques in Food and Agriculture, IAEA (CRP No.: D4.20.16) Vienna, Austria. The funding bodies played no role in the design of the study and collection, analysis, and interpretation of data and in writing the manuscript.

\section{Availability of data and materials}

The datasets, sequencing data and materials supporting the conclusions of this article are described within the article and its additional file, and materials will be provided by contacting the corresponding author.

\section{Ethics approval and consent to participate}

Not applicable.

\section{Consent for publication}

Not applicable.

\section{Competing interests}

The authors declare that they have no competing interests.

\section{Author details}

'USDA/ARS, Center for Medical, Agricultural and Veterinary Entomology, 1700 SW 23rd Drive, Gainesville, FL 32608, USA. ²Department of Insect Biotechnology in Plant Protection, Justus-Liebig University Gießen, Winchesterstr. 2, 35394 Gießen, Germany.

\section{Published: 18 December 2020}

\section{References}

1. Rubin GM, Spradling AC. Genetic transformation of Drosophila with transposable element vectors. Science. 1982;218(4570):348-53.

2. Paix A, Folkmann A, Goldman DH, Kulaga H, Grzelak MJ, Rasoloson D, et al. Precision genome editing using synthesis-dependent repair of Cas9induced DNA breaks. Proc Natl Acad Sci U S A. 2017;114(50):E10745-54.

3. Handler AM, O'Brochta DA. Transposable elements for insect transformation. London: Academic; 2012

4. Medhora M, Maruyama K, Hartl DL. Molecular and functional analysis of the mariner mutator element Mos1 in drosophila. Genetics. 1991;128(2):311-8.

5. Coates CJ, Jasinskiene N, Miyashiro L, James AA. Mariner transposition and transformation of the yellow fever mosquito, Aedes aegypti. Proc Natl Acad Sci U S A. 1998;95(7):3748-51.

6. Franz G, Loukeris TG, Dialektaki G, Thompson CR, Savakis C. Mobile Minos elements from Drosophila hydei encode a two-exon transposase with similarity to the paired DNA-binding domain. Proc Natl Acad Sci U S A. 1994;91(11):4746-50.

7. Loukeris TG, Livadaras I, Arca B, Zabalou S, Savakis C. Gene transfer into the medfly, Ceratitis capitata, with a Drosophila hydei transposable element. Science. 1995;270(5244):2002-5.

8. Fraser MJ, Ciszczon T, Elick T, Bauser C. Precise excision of TTAA-specific lepidopteran transposons piggyBac (IFP2) and tagalong (TFP3) from the baculovirus genome in cell lines from two species of Lepidoptera. Insect Mol Biol. 1996;5(2):141-51.
9. Handler AM, McCombs SD, Fraser MJ, Saul SH. The lepidopteran transposon vector, piggyBac, mediates germ-line transformation in the Mediterranean fruit fly. Proc Natl Acad Sci U S A. 1998;95(13):7520-5.

10. Warren WD, Atkinson PW, O'Brochta DA. The Hermes transposable element from the house fly, Musca domestica, is a short inverted repeat-type element of the hobo, Ac, and Tam3 (hAT) element family. Genet Res. 1994; 64(2):87-97.

11. Jasinskiene N, Coates CJ, Benedict MQ, Cornel AJ, Rafferty CS, James AA, et al. Stable transformation of the yellow fever mosquito, Aedes aegypti, with the Hermes element from the housefly. Proc Natl Acad Sci U S A. 1998; 95(7):3743-7.

12. Atkinson P. hAT Transposable Elements. In: Mobile DNA III. Washington, DC; ASM Press; 2015. p. 775-802. https://doi.org/10.1128/microbiolspec.MDNA30054-2014.

13. Handler AM, Gomez SP. A new hobo, Ac, Tam3 transposable element, hopper, from Bactrocera dorsalis is distantly related to hobo and Ac. Gene. 1997:185(1):133-5.

14. Handler AM. Isolation and analysis of a new hopper hAT transposon from the Bactrocera dorsalis white eye strain. Genetica. 2003;118(1):17-24.

15. McCombs SD, Saul SH. Linkage analysis of five new genetic markers of the oriental fruit fly, Bactrocera dorsalis (Diptera: Tephritidae). J Hered. 1992;83(3):199-203.

16. Handler AM, Gomez SP. The hobo transposable element excises and has related elements in tephritid species. Genetics. 1996;143(3):1339-47.

17. Lis JT, Simon JA, Sutton CA. New heat shock puffs and beta-galactosidase activity resulting from transformation of Drosophila with an hsp70-lacZ hybrid gene. Cell. 1983;35(2 Pt 1):403-10.

18. Bevis BJ, Glick BS. Rapidly maturing variants of the Discosoma red fluorescent protein (DsRed). Nat Biotechnol. 2002;20(1):83-7.

19. Riedel F, Vorkel D, Eaton S. Megalin-dependent yellow endocytosis restricts melanization in the Drosophila cuticle. Development. 2011; 138(1):149-58.

20. Finkelstein R, Smouse D, Capaci TM, Spradling AC, Perrimon N. The orthodenticle gene encodes a novel homeo domain protein involved in the development of the Drosophila nervous system and ocellar visual structures. Genes Dev. 1990;4(9):1516-27.

21. Vandendries ER, Johnson D, Reinke R. orthodenticle is required for photoreceptor cell development in the Drosophila eye. Dev Biol. 1996; 173(1):243-55.

22. Guimond N, Bideshi DK, Pinkerton AC, Atkinson PW, O'Brochta DA. Patterns of Hermes transposition in Drosophila melanogaster. Mol Genet Genomics. 2003;268(6):779-90.

23. Reid W, Pilitt K, Alford R, Cervantes-Medina A, Yu H, Aluvihare C, et al. An Anopheles stephensi promoter-trap: augmenting genome annotation and functional genomics. G3 (Bethesda). 2018;8(10):3119-30.

24. Horn C, Offen N, Nystedt S, Hacker U, Wimmer EA. piggyBac-based insertional mutagenesis and enhancer detection as a tool for functional insect genomics. Genetics. 2003;163(2):647-61.

25. Jarvik JW, Adler SA, Telmer CA, Subramaniam V, Lopez AJ. CD-tagging: a new approach to gene and protein discovery and analysis. Biotechniques. 1996;20(5):896-904.

26. Singari S, Javeed N, Tardi NJ, Marada S, Carlson JC, Kirk S, et al. Inducible protein traps with dominant phenotypes for functional analysis of the Drosophila genome. Genetics. 2014;196(1):91-105.

27. Palavesam A, Esnault C, O'Brochta DA. Post-integration silencing of piggyBaC transposable elements in Aedes aegypti. PLoS One. 2013:8(7):e68454.

28. O'Brochta DA, Sethuraman N, Wilson R, Hice RH, Pinkerton AC, Levesque CS, et al. Gene vector and transposable element behavior in mosquitoes. J Exp Biol. 2003;206(Pt 21):3823-34.

29. Handler AM. Prospects for using genetic transformation for improved SIT and new biocontrol methods. Genetica. 2002;116(1):137-49.

30. Bier E, Harrison MM, O'Connor-Giles KM, Wildonger J. Advances in engineering the Fly genome with the CRISPR-Cas system. Genetics. 2018; 208(1):1-18

31. Ren X, Sun J, Housden BE, Hu Y, Roesel C, Lin S, et al. Optimized gene editing technology for Drosophila melanogaster using germ line-specific Cas9. Proc Natl Acad Sci U S A. 2013;110(47):19012-7.

32. Serrato-Capuchina A, Matute DR. The role of transposable elements in speciation. Genes (Basel). 2018;9(5):254.

33. Handler AM, Zimowska GJ, Armstrong KF. Highly similar piggyBac elements in Bactrocera that share a common lineage with elements in noctuid moths. Insect Mol Biol. 2008;17(4):387-93. 
34. Hu J, Zhang JL, Nardi F, Zhang RJ. Population genetic structure of the melon fly, Bactrocera cucurbitae (Diptera: Tephritidae), from China and Southeast Asia. Genetica. 2008;134(3):319-24.

35. Roberts DB. Drosophila: a practical approach. Oxford: IRL Press; 1986.

36. Handler AM, Harrell RA. Germline transformation of Drosophila melanogaster with the piggyBac transposon vector. Insect Mol Biol. 1999;8(4):449-57.

37. Handler AM, Harrell RA. Transformation of the Caribbean fruit fly, Anastrepha suspensa, with a piggyBac vector marked with polyubiquitin-regulated GFP. Insect Biochem Mol Biol. 2001;31(2):199-205.

38. Pirrotta V, Steller H, Bozzetti MP. Multiple upstream regulatory elements control the expression of the Drosophila white gene. EMBO J. 1985;4(13A): $3501-8$.

39. Liu YG, Whittier RF. Thermal asymmetric interlaced PCR: automatable amplification and sequencing of insert end fragments from P1 and YAC clones for chromosome walking. Genomics. 1995;25(3):674-81.

\section{Publisher's Note}

Springer Nature remains neutral with regard to jurisdictional claims in published maps and institutional affiliations.

Ready to submit your research? Choose BMC and benefit from:

- fast, convenient online submission

- thorough peer review by experienced researchers in your field

- rapid publication on acceptance

- support for research data, including large and complex data types

- gold Open Access which fosters wider collaboration and increased citations

- maximum visibility for your research: over $100 \mathrm{M}$ website views per year

At BMC, research is always in progress.

Learn more biomedcentral.com/submissions 